\title{
SMART Technologies as the Innovative Way of Development and the Answer to Challenges of Modern Time
}

\author{
Natalia V. Vinogradova ${ }^{1}$, Tatyana N. Popova ${ }^{1}$, Abdellah Chehri $^{2}$, and Valentina I. \\ Burenina $^{3 *}$ \\ ${ }^{1}$ Togliatti State University, Belorusskaya str., 14, 445020, Togliatti, Russia \\ ${ }^{2}$ University of Quebec in Chicoutimi. 555, boul. de l’Université, G7H 2B1, Chicoutimi (Québec), \\ Canada \\ ${ }^{3}$ Bauman Moscow State Technical University, 2nd Baumanskaya str., 5/1, 105005, Moscow, Russia
}

\begin{abstract}
One of the promising tasks in education lies in reforming it into the knowledge economy, integrating and creating a market oriented towards results of intellectual activity. On the other hand, globalization process requires transition of the educational environment to the format of information, communication and digital space. Primarily these areas are set as the nodal tasks, which directs authors of this article to the comparative analysis of educational system making it possible to identify general and particular, positive or negative consequences and characteristics of digitalization in the higher education system. In accordance with current trends and processes of globalization and informatization, the authors are considering the prospects for interaction and mutual influence of Smart technologies used in building a future educational model in the higher education area. Technological innovations today are called upon not only to qualitatively change methods, forms and technologies in the education content, but rather to train personnel capable of operating in the new information and telecommunication community. Therefore, studying the influence and the capabilities of modern digital technologies that meet needs of society, on the one hand, and, on the other hand, contribute to formation of professional competencies in students, which requires major alterations in the learning process, changes in its state towards flexibility, adaptation, personalization, continuity, multidimensionality and systematicity, becomes of specific relevance for authors of this article.
\end{abstract}

\section{Introduction}

State crisis and further reconfiguration of political structures entails changes in social, technological and economic cluster, affects all spheres of life, including scientific and educational activities, creates prerequisites for the development of new social processes, develops culture and art, as well as promotes generation of a new information society. Instability in social, economic, political and cultural development of the country indicates

* Corresponding author: bvi@bmstu.ru 
the necessity primarily in the educational sphere to identify ways to overcome global crisis by the state and determines tasks aimed at attracting and developing the intellectual resource as a strategic factor in the innovative improvement of production, science and technologies.

Higher education is becoming a moving force in formation of a society and of an economically developed country, but at the same time it requires appropriate personnel both engineering and pedagogical, who would be focused on design, development and implementation of new technologies and innovations.

The problems faced by higher education are caused primarily by the transition to technological post-industrial way of life, which requires a person to understand and ensure conscious use of natural, ecological, economic and technological resources, but also of human capital, as human life and continued existence on the planet are the main tasks and values of a society.

Education is not only a special environment. Its specific results are being developed, supplemented and changed in accordance with the market and social requirements. Thus, industrial society needed education that contributed to the development of mass communication, formation of a certain quality of human life, industry, economy and labor productivity. Such education, as a rule, is called mass or "classic". During that period, the main form of knowledge transfer was lectures, books, tutor experience, his recommendations in studying the material. The result of academic education was to obtain a specific specialty, i.e. profession.

Continuity of education and generation of competencies in the 21 st century information society led to the use of remote, electronic, remote access on-line training implemented using telecommunication technologies and characterized by indirect interaction between the tutor and the students. Naturally, such educational process differs from the "classic" one in its visual expression, attractiveness, accessibility and mobility.

In accordance with this, the developed e-learning system expects introduction of information databases, which technical means and technologies provide transmission of necessary information via the Internet communication lines. This, in turn, requires not only the development of new pedagogical approaches, means and methods, tools of influence, but also organizational changes, personnel competence, regulatory basis, material and technical equipment and methodological improvement.

On the one hand, using digital technologies and Internet services makes it possible to obtain new effective advantages that improve human life, lead to creation of a new SMART society, where there are only smart technologies, cities and state, business, economy, infrastructure and the individual himself, as well as to generation of new SMART culture, consciousness, thinking, literacy and environment.

With the IT development, transformation of a person occurred, and primarily in his thinking and perception. The current Generation $\mathrm{Z}$ has no boundaries between the virtual and the real worlds; for them Internet is the natural component in interacting with the world and society. Therefore, they have completely different priorities, ideals and perception of the world, they think differently in categories of relations, family and society.

For such digital society, where Generation $\mathrm{Z}$ is staying, learning through the Internet becomes the way to join this community and the way of self-identification there. The main resource is not a plant, but a person, his intellectual resource, his abilities and competences in creative implementation of task set by means of IT. Thus, the "classic" education no longer meets the requirements of Generation $\mathrm{Z}$, which lives in the world of physics + digital. Already now it could be noted that compared with 2013 the students' on-line activity increased by 2.5 times; and this is comparable to the fact that about $53 \%$ of adolescents spend 8 hours a day in the Internet. It should be understood that this is an entirely different community of people, whose process of learning and receiving 
information is built on the principles of personalization or on individual trajectory and interests. Against the background of such educational system digital improvement, completely new competencies and new consciousness appear, owning which an individual would be able to live and work in conditions of uncertainty. Information society connected to SMART technologies is characterized by the increased amount of e-information and eservices using these technologies, which requires mobility, continuity of education, focus on self-education and readiness for retraining from this generation.

As a result, previously used technologies were based on knowledge and information that were transformed into technologies aimed at exchange of experience; and SMART technologies are introducing significant changes in strategy and tactics of managing the educational process. This will subsequently lead to the fact that society would require specialists having skills in working with electronic databases and sources. This is the new philosophy of SMART education, where training is based on the use of mobile content available worldwide and providing free access. Such smart, flexible and mobile education is built on integration of various educational institutions and tutors all around the world engaged in the development of new standards, technologies and educational contents in implementing unified combined education and online activities. The meaning of SMART education lies in the availability of knowledge expanding both temporal and spatial boundaries of learning and located in a unified repository of the intelligent search system.

Thus, paying special attention to the quality of education, introduction of new educational technologies, development of new training programs, it is necessary to solve the problem of generating an efficient, innovative system that ensures its functioning and improvement of its constituent elements. By the innovative system, the authors understand such modernization processes in the content of education, in the structure of educational modules, in the mechanisms of education management, in the technologies of teaching and educational processes, methods and means of training and education that ensure functioning of the educational sphere, its organization, management, direction and control.

The purpose of this article is to identify and justify modern areas in education, such as development of cloud technologies and distance learning. At the level of modern technologies and concepts in the modular competence-based learning model connected to informatization and digitalization, educational environment, technologies and teaching methods are being modernized; here Smart education acts as a means of technical and social development of society and person [1], which involves creation of modern information and education infrastructure, development by students in real online (offline) mode of new means of communication that provide mobility, activity and accessibility in their life and learning.

At the same time, it should be understood that SMART education possesses its own culture and philosophy of knowledge, which requires a completely different approach to education. The authors analyze SMART technology as a technological innovation in reforming the training system capable of creating "their proprietary education" in the postindustrial society corresponding to the digital progress in society and "smart economy". Internet in the modern world became a special reality, habitat and communication space, but also the space that generates network identity. Accordingly, Internet today is not only a means of storing and transmitting information, but also a special form that generates new activities, cultures, meanings and concepts.

The authors are considering SMART technologies as one of the prospects in creating educational platform, intellectual environment, as a new approach to education and are studying the impact of these technologies on the student personality development in the context of modern trends using modular competence-based approach to education. 


\section{Smart technologies value content in higher education system}

Globalization becomes in the XXI century the key process determining development of the world; and it lies in creating a system of unified world-wide information service and platform. The process of global informatization system that penetrated not only into economy, politics, but also into culture and education, is associated with integration and unification of world information. Digitalization is the main form of such transformation in the education system, which appears to be the translation of information into units and zeros. On the one hand, information and communication technologies are paying particular attention to the quality of education in the context of the educational process and are contributing to creating a unified information infrastructure and educational platform, which provided universities with new distance learning technologies in the transfer of knowledge and data. On the other hand, it contributed to the development of information and communication competence among students. Information accessibility and openness of the learning system allowed students to determine their educational trajectory themselves $[2,3]$. Following this, new technologies resulted into new mechanisms, programs, forms and methods aimed at creating a new type of personality, new personnel, who having formed these skills in themselves would be able to develop them in those, whom they will be teaching in the future. This means that changes are necessary not only in types of activities, competencies, but also in the style of thinking and perception.

Nowadays, Internet is a new reality, main habitat, space for learning, education and human development. IT became an integral part of life in the modern society, where elearning is being replaced by SMART education, and later by SMART technology. Currently, there are no clear notions of the SMART technology concept; and most publications on this topic are providing futurological definitions associated with changes in the education system, which causes many to have conceptual uncertainty and incomprehensibility of this educational paradigm.

SMART is connected to various achievements in information technology. Accordingly, its main function lies in creating an educational environment that is changed through digital servers.

SMART technologies formation was influenced by technologies transition from Web 2.0 to Web 3.0, as well as by social servers, such as Facebook, Twitter, YouTube video hosting, Google blogs and services, Wiki website that make it possible to create proprietary information sites and various content. At the same time, these technologies perfectly fit into the educational space and became the main means of interaction in the feedback between tutors and students making the learning process enthralling, mobile and flexible.

SMART Education term appeared against the background of post-industrialism and the country technical development and was not accidental, but rather it reflects those changes that began in the society of competencies and its informatization. These changes are based on their own logical chain in application of new digital technologies. If we analyze those key competencies that a graduate should possess, and we rely not only on the FSES, we should rather proceed from the fact, what knowledge and skills a graduate should possess and what he would need in future activities in the digital economy. The learning process is laid on the shoulders of a tutor, who develops plan, content and strategy of the educational area and, first of all, forms such competencies that would correspond to the social and economic development of the 21 st century society. Then, speed of information, its relevance, science linkage, importance and its accessibility along with the knowledge and technology side are becoming the important components of education. And the result of such training will be a person, who has an intellectual resource valuable to society, with 
relevant capabilities and ability to work for the goods of the society and to be a citizen of the Russian Federation.

So, what are the key knowledge and skills, competencies of the 21 st century that could lead to the development of intranational and national global economic, political and cultural systems? It is not the availability of knowledge amount that comes to the fore, but the level and type of thinking.

When applying for a job, a specialist is estimated by his ability to perceive objects and phenomena holistically demonstrating the ability to identify patterns in various processes that led to changes, i.e. the employer evaluates him by the presence and type of systemic thinking. Collecting information in pieces, is he able predict the results, solve quickly emerging problems, build a logical chain of these key processes that affect the development of social and economic sector of the country and society.

SMART technologies consider education as an exciting process, which paradigm is lifelong learning. Information and communication components contribute here to development and formation of interdisciplinary communication among students. It is safe to say that today it is not enough to know, be able to and own a single profession. Specialists are required, who understand several areas at the same time. For example, already now employers representing many social and industrial areas demand knowledge from their subordinates not only within their professional area, but also at the junction of two areas. Higher education system curricula are built on the basis of two profiles. Thus, one of the future sought-after professions is the HR manager, who knows how to work with IT systems, mathematics and basics of personnel management. And these requirements will only grow every year.

Another competence necessary in the future follows from this. Ability to create, organize, manage and control processes and projects. Moreover, this competence is considered as the ability to manage project activities both within the organization and on remote access. However, it is not the main type of professional activity. Important quality of a personality assessment will be his understanding of principles and approaches in the processes and projects management.

One of the main skills of a specialist is his ability to work with various IT systems. Thus, large companies and enterprises with significant working capital (for example, the Russian company Lukoil) are working with SAP systems, and smaller ones - with 1C. SAP system is software that makes it possible to systematize information processing in many departments of the organization relating to financials, monitoring, personnel, trade transactions and procurement. This skill would be mandatory for everyone.

Customer focus and working both as a team and with people are the next key competences in the 21 st century in the corporate training area, on the one hand, and, on the other, serve as an index of measuring the net promoter score (NPS). Many companies create coworking centers within their enterprises as a space flexible enough and able to unite various groups and communities with their proprietary internal culture. Main task of such centers is the ability to work, communicate correctly, efficiently and quickly, not only with internal, but also with external customers. Teamwork and empathy skills should ensure development of feelings and ability to listen and hear each other in employees.

Awareness as a competence is determined by the continuity in monitoring situations that occur with a person and is defined as the ability of thinking in regard to introspecting ongoing activities. It makes the employee a valuable specialist, as it makes it possible to listen to oneself, helps to concentrate on a situation providing balance and harmony in the internal state.

Competences that developed during the predictable SPOD in the world in the digital age form completely different skills, these are the skills to work in a VUCA world, i.e. in situations of volatility, uncertainty, complexity and ambiguity, where the world is 
constantly and rapidly changing. This, in turn, requires the person to quickly process enormous amount of information and to ensure its adequate estimation. When working with enormous amount of data, we sort, discard and forget it. Such people should possess high level of intelligence, systemic, creative and non-standard thinking, flexible mind, adequate self-esteem, tolerance and indulgence, ability to quickly analyze and vary solutions.

Facebook, Google and Twitter are the companies guided by the Diversity principle based on openness, multiculturalism and inclusion into the personnel policy, which makes it possible to structure business with people of different appearance, age, gender, nationality and sometimes of different culture and country. Only in such a community, as many leaders of large companies believe, alternative solutions are appearing, as people are learning to be flexible, becoming able to listen more carefully, finding a language to speak with others and understanding a different point of view, which requires an open mind and guarantees realization of equal opportunities for everyone.

Communication is one other important competence in business, culture, education and economics. We communicate via Internet with a large number of people and various communities. Education as well as business became an open issue, which clearly requires moving forward, creating new contacts and new content, exchanging the ideas. At the same time, Internet has its own means of communication, goals and objectives, tools that could convey information, thoughts to a contact with remote access and, at the same time, evaluate each moment of communication as a kind of currency of attention in the modern digital communication era.

The above competencies became a key driver in the development of a digital society, its needs and requirements to the educational system providing specialists ready to work and develop.

Transition from e-learning to Smart Education involves creating such an educational concept, which is characterized by flexibility, diversification, mobility, ability to change, adaptation to needs of society and demands of the labor market.

In this regard, SMART Education concept is comprehensive modernization of the educational space, entails emergence of such technologies that make it possible to produce educational content leading to creation of a holistic educational ecosystem accompanied by continuous education [4]. It should be understood that the subject is creating the global educational content, including creation of an educational company [5], that provides training using a common repository of educational materials, where all knowledge is built on the principle of integration and is connected to a metadata system. And these data could be transformed, changed and amended, and on their basis various forums, training seminars and on-line courses could be created.

SMART education development today involves creation of highly intelligent smart and high-tech environment, convenient and comfortable, where development of information and communication competences among students and development of modern IT is taking place. Its objective is to make learning effective by introducing various computer programs and multimedia applications, SMART devices (SMART board) in the electronic media making it possible to create a virtual learning environment with unlimited possibilities for students. With availability of such education, it would become individually oriented, continuous, permanent, included in a student life everywhere and always.

Trying to give a constructive definition of SMART technologies in the higher education space, we undoubtedly agree that these technologies are aimed at advanced learning allowing, first of all, to use social, information and communication tools and innovative technologies in order to increase the level of professionalism, expand possibilities in personal development, make the learning process dynamic, interactive, visual, bring the quality of educational services to the new level of knowledge, outpacing production and technology development that meets requirements of digital economy, labor market demands 
and needs of society. And this means that this type of education should have its status, its content and technologies ensuring interaction between a tutor and a student with the use of social tools and IT in the educational process.

\section{Negative risks and positive effects of smart education}

Traditional form of education today is inferior to active forms of education. This is primarily due to the involvement of students in the cognition process. Many studies show that with active perception information is stored in memory in the amount of about $80 \%$, and about $90 \%$, if this information is obtained on what was done by ourselves, which means that material assimilation occurs through motor skills, interactive participation, auditory and visual concentration. Now it is becoming possible, since SMART education as the educational environment has a wide line of interactive interaction, is built on special forums, lectures, dialogs with artificial intelligence. As a modern trend, SMART education, on the one hand, has certain technological advantages in the form of accessibility, mobility, personalization, flexibility, education openness and stands for: S -self-governing, selfcontrolled, M - motivational, A - adaptive, R - variative, T - technological education. Such smart learning implies creation of educational platform, active content, which involves combining university with tutor and students in order to implement the educational process. On the other hand, today's distance on-line (e-learning) education separated from its ideological, meaningful and semantic component, became a spectator and a consumer service. Several factors should be noted.

Distance on-line education could lead to certain risks, which is caused not only by external, but also by internal circumstances. However, linking creation of a unified information and educational platform with SMART technologies, within which framework a certain technological, instrumental, partner and documentary space is being created, there also appear difficulties that today are becoming fundamental. Thus, digitalization in education dislodges real educational institutions replacing them with virtual, on-line education. This leads gradually to primitivizing competences. Electronic assistants, navigators, helpers and other software programs are atrophying many competencies; in particular, they reduce incentives for independent search for information and aggregation of knowledge, which manifests itself as a part-to-whole problem. This could lead to disruptive selection tearing the education system into the following: generators, i.e. specialists able to generate a new picture of the world and to create new knowledge, and operators, who know, which software product could be used to find the answer to a question. It is important also to take into account that changing the role of electronic assistants would lead to the fact that they are becoming the central link in specialist training, which might affect education of generators.

Another risk is connected to the fact that already now the number of correspondence and distance learning students is increasing. As for further education, only $16 \%$ of the Russian population is involved in this process, while abroad the number ranges from 45 to $60 \%$. We see a decrease in the quality of education and its imitation. It will also lead to a change in the role of tutors, as the tutor could be replaced either by artificial intelligence, or the tutor would become a curator or a tutor contacted only if necessary. Namely, the educational development vector depends on the teaching staff, whose main activity is improving technologies and curricula, developing a fund of estimation tools in accordance with types of professional activities aimed at developing specific set of competencies. Formation of the competitive services market in the educational area is built on the basis of those professional competencies that graduates possess; and these are primarily skills in project management, ability to solve various problems in the educational process management and organization using various means, forms and technologies. 
This trend has a probable negative result associated with the possibility of probable damage; but we are meaning the spiritual component. We understand that for society, economy and culture development a new graduate model is required, where a graduate acts as an intellectual product. The state should allocate funding for this, which in the future would help to change the educational environment, and also to join the socium global information space.

Digital world is becoming the modern value in post-industrial society. Despite its positive aspects in the educational processes technologization, which consist in the knowledge transfer, they could lead to false competences, when ability to find the required information is equated to formation of professional competences among students. From the point of view of SMART characteristics, such technologies make it possible to choose the trajectory of their education by adults, apply individual schedules and implement fairly close relationship not only between tutor and students, but also with future employers, where training efficiency is measured by the level of updated competencies.

In order a student could master such competencies, it is necessary to change the content of education, its methods, tools and technologies. It should also be understood that a tutor must self-develop along with the students and take several steps ahead. Is a tutor of today ready for this? Is a tutor experienced in SMART technologies? Was there any kind of SMART training? How could these forms be used in professional activities and in humanitarian or technical education areas?

SMART technologies present a tutor with new challenges in education. A tutor must possess not only knowledge and significant amount of information in the professional area, but also should be able to use various resources in working with students. Opening up new opportunities for the tutor being the author - developer of a personalized course or content, he should learn functions of a leader, organizer, partner, service provider and process controller. Two questions arise. One leads to normative and legal use of intellectual property. That means, how rights of the content author would appear in the unified education and information community? Who will be the copyright holder of unique educational content? The second is looking for an answer to the question, when a tutor should practice sciences. The question is not only in saving time. Tutor work is iterative in nature, as the content is being constantly updated, amended and changed, which in essence is a long-term process and will always have the status of "the content is in the process of updating and editing".

One of the possible SMART technologies disadvantages is the reduced role of tutors in the context of direct interaction with students, where personal influence of a tutor on the student is missing. Such conceptual changes in education could lead to formation of technocratic and engineering thinking in the latter and to students' inability to examine the world in its natural, physical, cultural, emotional, value, ecological integrity and uniqueness.

Education became a source of new training relationships, including knowledge, technology and innovation markets. "Educational paradigm transformation led to changes in values, meanings, goals, educational programs, technologies, changes in the structure of society and the worldview of a person" [6]. The same tasks are determined for artificial intelligence, and forecast how digital technologies would affect the development of society and man. Gadgets and global network primarily reduce the level of socialization in a person $[7,8]$. Fine hand motor skills, working with keyboard and with electronic tablets also affect vision, cause tension and fatigue of the eyeball. And that ultimately could affect the mastery of competencies [9]. Lectures and visualizations are used in higher education [10], which are also demonstrated with information technology.

Prolonged use of the keyboard could lead to physiological changes in fingers, shoulder girdle, joints and muscles. Today, monitoring condition of schoolchildren and adults, office 
workers and those who spend most of their working days in a sitting position at a computer or with a gadget makes psychologists and doctors sound the alarm. Such a lifestyle, lack of load on the musculoskeletal system or when the load is only on a certain body area (skeleton) could lead to hypokinesia. This is manifested in such pathological processes as osteochondrosis, osteoporosis and metabolic syndrome. Constant change of information on a blinking screen leads to changes in the brain, which reduces cognitive activity, and radio frequencies that are emitted by gadgets could lead to possible serious diseases.

One of the possible negative effects, as we also see, is that a personal file is created for each person, detailed information about the family is collected, and a characteristic is compiled. In addition, when developing educational contents and placing them in the digitally accessible global network, it is necessary to consider not only the information and communication accessibility system, but also the information security and reliability system. Smart education requires regulated actions by the public sector, normative and legal sphere related to digital literacy, transparency of information, and network personality. This problem raises issues affecting improvement of the education system in order to raise the level of digital literacy in both students and tutors. It could also provoke appearance in the electronic educational environment of content with poor-quality information. We see this aspect of the problem in digital control of information and in its subjectivity. But we do not muse that a student would think inventively $[11,12]$, be creative $[13,14]$ and explore the scientific world, if modern technologies are provided.

In order to post content on the global educational platform, it is necessary to introduce a system of special filters and an expertise, which review would present a positive or negative result. There appears a serios risk that all this could lead to replacing the surrounding world with an artificial world of information technologies and to extruding natural sensitivity and humanistic culture from a person's life [15], destroying direct genetic connection between the world and a person [16].

Another factor of a possible to some extent fantastic risk to the individual is total monitoring. On the one hand, a person has the right to privacy, and on the other, all his actions would be registered with a video camera. Cell phones are equipped with electronic sensors with the GPS tracking system that makes it possible to find a person and to recognize him by individual signs. Camera allows not only tracking personal life, interests, hobbies, movements, travels, follow a page in the network and collect information about an individual. This provides the state and various companies with a powerful tool in controlling citizens. China especially advanced in this development, where in some cities total video control over the population was introduced, and this basis scoring is assigned to a person. Such a system of total control would not be appreciated by everybody. Perhaps, there will be hackers, who would crack the tracking system, which will require special information protection for a person, development of powerful software programs, computers and on-line processing. Against this background, Neuronet or WEB 4.0 ideas are being developed, which essence is that development of the World Wide Web would be based on interaction of all participants, both humans and animals, on the principles of neuro communication. In this regard, forecasts are appearing, and hypotheses indicate world technological development towards its uncontrollability, against the background of which a superintelligence would be created that surpasses the entire humanity.

Despite such fantastic forecasts, it is safe to note that SMART technologies generate new consciousness, new status and new human competencies, new qualifications and specialties. Here, the boundaries of property and employment are being blurred. This opens opportunities for interacting in communication, while personification of a person occurring with the Internet has its own virtual space, its own culture and logic of using information, its own structures in their interaction. On the other hand, these data indicate now that against the background of SMART technologies there appears a need to analyze 
information that creates new technologies, i.e. new methodology in science, education, economy and production.

However, SMART education positive aspects could be highlighted among other things, which is primarily associated with improvements in digital Internet technologies, development of interactive forms, wireless technologies such as $\mathrm{Wi}-\mathrm{Fi}, 5 \mathrm{G}$ cellular communications; and, according to the Tomorrow Cloudy scientific journal, energyefficient long-range networks, software-determined radio, development and implementation of a vehicle connected to everything and much more will soon appear.

As for the use of SMART technologies in the educational process, here educational space in such disciplines as history and philosophy, project activities, research work in 3D reality is being amended and expanded; and it is possible to form not only professional competencies, but also to lay patriotic content. However, it should be understood that this is a very time-consuming process that requires special attention to it, as it is associated both with psyche, thinking of a person and with his culture.

In addition, SMART makes it possible to use the network and digital technologies in creating various communities and forums, disseminate not only information and create educational material, but also to exchange professional experience and opinion enriching the content-educational component of education. Then, communication between tutors, students and parents becomes equal. Each student, when mastering a program, would be able to take a course along an individual trajectory or program depending on personal pace of work and independently of the others.

Such technologies are providing for interesting, original and visual approach to seminars and lectures. For example, a recent seminar on the history of Russia in the XX century at Togliatti State University allowed students to divide into groups, study various issues of culture, industry, economy and to present a fancy-dress report at the seminar, which main idea was to discuss various and important topics that the country was living with at that time and to come to a definite conclusion in order to further develop skills of system analysis and holistic view on that epoch revealing political, economic and spiritual life of the society, as well as to clue the entire diversity of that period cultural heritage. This fancy-dress report was only possible due to SMART technologies introduction.

\section{Conclusion}

The module competence-based approach is aimed at creating competencies, providing quality training for a specialist, who has deep fundamental and essential knowledge, is able to simulate and modernize the future, and on the other hand, is able not only to design, but also to manage this future for the benefit of society, nature and people. Education is not a series of training exercises necessary to master the competencies. Education is a space with a certain system of internal meaning aimed at deep understanding and comprehension of the essence of phenomena and concepts of human life and space, art, culture, science and traditions. Education being a spiritual and intellectual value combines professional activity and is becoming that sense generating and vital integrity introducing interconnection between nature and people, science and practice, society and culture. This is an incessant search based on the platform of deep and fundamental knowledge. Ultimately, society development is characterized by one criterion, whether a person enriched the culture of people, society, tradition, and how it is possible to enrich what you do not know.

Thus, we could speak of a deeper sense of education, as a space having systemic, holistic, multidimensional and multi aspect nature of activity considered as a valuesemantic strategic form on a par with technological and applied activity, where educational and learning spheres are under mutual influence and interaction between society and surrounding world, science, creativity and practice, where education affects the 
development of thinking, memory, perception, speech, and goes in two aspects, i.e. general culture and professionalism.

Introducing Smart technologies in education, we are facing the need to search for certain means and relationships among a variety of courses, modules and content aimed at creating new information and sociocultural space. Lifelong Learning principle proclaimed by UNESCO very accurately expresses the Smart education position. These technologies act as the means of creating intellectual virtual educational environment, which is distinguished by its accessibility and information, expanding the tutor ability in selecting the knowledge transfer tools. Such technology is efficient, when it is part of the educational process and is not replacing it. On the negative side, we see that Smart technologies are kind of a Play Market, which helps to install any application offering a wide range of education categories. Just enter the name of an academic subject, and a list of mobile applications for any discipline, in English and Russian, history, literature, algebra, chemistry, biology and art would appear. Will this transition adequately close all levels of educational development and interaction, maintain the tutor feedback with a student? Indeed, educational goals are achieved in the aspect of expanding the student's range of vision, increasing their level of general culture and education, as well as their culture of thinking, communication and speech [17].

SMART education possesses naturally important practical and theoretical significance in management, design, technological, methodological, scientific and research activities of a university seeking to maintain its position in the education market in order to enter the global unified information space. Its objective is to attract foreign students and tutors in organizing foreign practices. This makes it possible to implement skills in relevant activities, develop and form creative abilities, research skills, leadership and management qualities, as a new way of thinking. And in this sense, in order to occupy its economic positions influencing or creating the market of society demands as a whole, HEI digitalization is becoming a key element in providing flexible and adaptive educational platform, competitive advantage for a university that could attract students and become a center of competence owing the intellectual capital. As the number of educational platforms and content users is growing, the HEI image is rising. However, high level of competition requires permanent development, improvement and updating of the educational programs. This includes the language barrier among students. Today, such digital service in the Russian HEIs is at the stage of its implementation, while abroad, it is the main element in education.

SMART education content side is expressed in fundamental differences, in the education structural component and in possessing the basic information services, which would lead to qualitative changes in the process of interaction between tutor and students. Different and multivariate technologies, means of training and education would be manifested both in objectives and mechanisms of influence on the learning object. Consequently, the result would be seen in a new model of innovative graduate and formed competences.

It should be noted that the Government Decree and the President's Message on the Strategy for Development of Electronic Industry in the Russian Federation until 2030 was published January 23, 2020, and it indicates that modern task of society and Russian education today is its sovereignty and, first of all, in regard to Internet, which means occupying the global technology leadership market. Objective of this strategy is expressed through the development of scientific, technical and human potential to create a competitive industry that develops new technologies, as well as the normative legal basis that meets requirements in modern electronic products.

In this regard, digitalization urgent tasks will include monitoring activities of all participants in the educational process, localizing openness within the country, rejecting the 
Bologna system, increasing budget and budget admission. And besides, creating proprietary electronic hardware. Banning anonymity in the network. Creating Russian analogues of the SCOPUS system with very strict rules for foreigners.

Thus, SMART technologies, which replaced e-learning, became an integral part of both the educational environment and the human life. They focused attention on what society and future education will look like, how these technologies would affect education development in general and further changes in this system.

Today's revolution, which was brought by SMART technologies, relates primarily to teaching methods and system for assessing the quality of knowledge [18]. It should be understood that most students and tutors are not using SMART technologies in their practices, because they do not know about them.

And a serious question regards certainly the use of SMART technologies in technical and humanitarian disciplines. How will these technologies influence and contribute to development and formation of a holistic and harmonious personality? What is one of the priority conceptual tasks of an education system? Where an educated person is a person who has his own beliefs and worldview built on deep knowledge, able to think critically, analyze and understand another person. Spiritual, moral, emotional, value oriented and patriotic components of education are very important in education. This necessitates formation of digital literacy among students, so that the next Generation Alpha would use SMART technologies confidently, efficiently and safely for all mankind.

SMART technologies within the concept of smart education raises a number of questions. The following could be selected among them.

How will unification of joint educational activities of tutor, educational institution and prospective employer be organized? Who will be the author of developed content? Where will it be stored? In public domain or will students be provided with a password to enter the cloud server? Developed educational content should have appropriate sources, information resources and links. What open or closed sources could be referenced and how could they be determined for compliance?

Flexible schedule in the learning process allows students to change a HEI and to select their own learning programs. Would such a system lead to the fact that a student without completing the course is moving to another institution leaving on his back the status of eternal student or staying with academic backlog? If a student has the right to move from one HEI to another, then who and how will control that? Which HEI will provide a document on obtaining the appropriate qualifications and a diploma of education? This, in turn, requires regulated actions, both from the standard and from the state.

On the other hand, by forbidding students to use capabilities of artificial intelligence, we primarily limit the area of action and thinking and are not taking into account the gender differences in students [19].

There is only one way out, it is necessary to develop individual educational trajectories that require other competences from students and their own unique set of knowledge, abilities and skills. SMART technologies independence, autonomy and seamlessness provides equal opportunities in education ensuring effectiveness not so much by acquired knowledge and competencies, but due to the opportunity to use them in practice. Once acquired education could no longer guarantee career growth, efficiency and professional activity in the future. A person could not be called a professional, if he is not improving and self-developing, if he does not update previously acquired knowledge, does not know how to analyze problem situations, and does not pose new tasks. Motivation and thinking are playing an important role [20].

Smart education concept lies in creating the intelligent friendly environment for continuous development of students' knowledge, skills and competencies in the interests of society and state. 
Thus, SMART technologies in the rapidly developing information community are the new Lifelong Learning educational paradigm, where IT potentialities are necessary as generation of new knowledge and new specialists ready to carry out social orders at various levels, promptly respond to innovations, quickly create and introduce innovative products into manufacture.

The fact remains evident that the need for a new system of smart digital SMART education is not accidental. So, SMART education in its concept involves creating such environment and space, where using content in free access would provide interactivity and learning mobility, which, however, is a rather difficult task to be integrated into today's higher education system.

Coming out of the current situation, we consider precisely technological opportunities among current innovations proposed by SMART technologies in building an intelligent environment based on a mixed form of training, in developing forums, lectures and on-line courses. Developed and implemented programs and their content should cover not only strategic, substantive, technological, tactical, operational, but also control, regulatory, emotional and creative levels. The modern world of information technologies is rapidly introducing its changes. It is important to elaborate a proprietary strategy reflecting not only specifics of the university, but also quality of education, what the higher education is proud of.

\section{References}

1. S.A. Gudkova, T.S. Yakusheva, A.A. Sherstobitova, V.I. Burenina, Modeling of scientific intercultural communication of the teaching staff at smart university (2019). DOI:10.1007/978-981-13-8260-4_48. Retrieved from www.scopus.com.

2. Y.S. Mitrofanova, A.A. Sherstobitova, O.A. Filippova, Modeling the assessment of definition of a smart university infrastructure development level (2019). DOI:10.1007/978-981-13-8260-4_50. Retrieved from www.scopus.com.

3. Y.S. Mitrofanova, A.A. Sherstobitova, O.A. Filippova, Modeling smart learning processes based on educational data mining tools (2019). DOI:10.1007/978-981-138260-4_49. Retrieved from www.scopus.com.

4. M.G. Sergeeva, N.N. Bedenko, L.Z. Karavanova, T.Y. Tsibizova, I.S. Samokhin, M.S. Mohammad Anwar, Educational company (technology): Peculiarities of its implementation in the system of professional education, Espacios, 39(2) (2018). Retrieved from www.scopus.com.

5. M.G. Sergeeva, N.N. Bedenko, T.Y. Tsibizova, M.S. Mohammad Anwar, T.G. Stanchuliak, Organisational economic mechanism of managing the growth of higher education services quality, Espacios, 39(21) (2018). Retrieved from www.scopus.com.

6. N.V. Vinogradova, G.M. Zemlyakova, Pedagogical engineering as generating scientific methodological in students of 44.04 .01 training direction, Pedagogical education, ANI: pedagogy and psychiatry 1(22), pp. 73-79 (2018).

7. A.O. Karpov, Education in the knowledge society: Genesis of concept and reality, International Journal of Environmental and Science Education, 11(17), pp. 9949-9958 (2016). Retrieved from www.scopus.com.

8. A.O. Karpov, Socialization for the knowledge society, International Journal of Environmental and Science Education, 11(10), pp. 3487-3496 (2016). Retrieved from www.scopus.com.

9. G.N. Taranosova, M.G. Lelyavskaya, T.S. Yakusheva, Text competency at higher school philology training, AstraSalvensis, T. 6, pp. 806-824 (2018). 
10. T.Y. Tsibizova, V.M. Postnikov, S.B. Spiridonov, Analysis of the impact of technology lectures-visualizations on the results of control measures in various academic disciplines, Perspektivy Naukii Obrazovania, 33(3), pp. 358-363 (2018). Retrieved from www.scopus.com.

11. A. Alizamar, A. Afdal, I. Ifdil, Z. Ardi, A. Ilyas, Z. Zikra, R.D. Febriani, Are there statistical anxiety differences between male and female students?, Paper presented at the Journal of Physics: Conference Series, 1157(4) (2019). DOI:10.1088/17426596/1157/4/042127. Retrieved from www.scopus.com.

12. A. Alizamar, A. Afdal, I. Ifdil, Y. Syahputra, Exploration of students' creativity based on demography, International Journal of Innovation, Creativity and Change, 5(1), pp. 50-65 (2019). Retrieved from www.scopus.com.

13. E.V. Endovitskaya, Formation of organizational culture that stimulates innovativeness and creativity of personnel (2020). DOI:10.1007/978-3-030-15160-7_88. Retrieved from www.scopus.com.

14. N.S. Schutte, J.M. Malouff, Connections between curiosity, flow and creativity, Personality and Individual Differences, 152 (2020). DOI:10.1016/j.paid.2019.109555.

15. S.A. Gudkova, T.S. Yakusheva, A.A. Sherstobitova, V.I. Burenina, Modeling, selection, and teaching staff training at higher school (2019). DOI:10.1007/978-98113-8260-4_54. Retrieved from www.scopus.com.

16. N.V. Vinogradova, A.V. Soroka, Problem of value and notional orientation in children artistic education system, World of science, culture, education, No. 3 (70), pp. 22-24 (2018).

17. T.N. Popova, Problem of forming professional foreign language communication in bachelor students within the frames of Foreign Languages discipline, Togliatti: TSU Publishing Hoise, pp. 117-123 (2018).

18. O.M. Shcherbakova, O.Yu. Otrokova, V.I. Burenina, Russian method of training engineering personnel: historical experience and modernity, Advances in Social Science, Education and Humanities Research Proceedings of the 4th International Conference on Contemporary Education, Social Sciences and Humanities (ICCESSH 2019), pp. 645-650 (2019).

19. A. Abraham, K. Thybusch, K. Pieritz, C. Hermann, Gender differences in creative thinking: Behavioral and fMRI findings, Brain Imaging and Behavior, 8(1), pp. 39-51 (2014). DOI:10.1007/s11682-013-9241-4.

20. T.N. Popova, O.V. Dolzhenko, Importance of language preparation in life of a future specialist, Actual problems of theoretical and applied linguistics and optimization of teaching foreign languages, International scientific extramural conference (October 67, 2016): information package / Corr. ed. Yu.I. Gorbunov. - Togliatti, TSU Publishing House, pp. 159-165 (2016). 